\title{
Overexpression of Avocado (Persea americana Mill.) PaRAP2.1 Promotes Fatty Acid Accumulation in Arabidopsis thaliana
}

\author{
Weihong $\mathrm{Ma}^{1}$, Xiaoping Zang ${ }^{1}$, Yuanzheng $\mathrm{Liu}^{1}$, Lixia Wang ${ }^{1}$, Jiashui Wang ${ }^{1}$, Yanxia $\mathrm{Li}^{1} \& \mathrm{Yu} \mathrm{Ge}^{1}$ \\ ${ }^{1}$ Haikou Experimental Station, Chinese Academy of Tropical Agricultural Sciences, Hainan, China \\ Correspondence: Yu Ge, Haikou Experimental Station, Chinese Academy of Tropical Agricultural Sciences, \\ Hainan, China. Tel: 86-0898-6677-0005. E-mail: geyu@catas.cn
}

Received: March 14, 2021

Accepted: April 15, $2021 \quad$ Online Published: May 15, 2021

doi:10.5539/jas.v13n6p1

URL: https://doi.org/10.5539/jas.v13n6p1

The research is financed by the Natural Science Foundation of Hainan Province of China (grant number 320RC683), Budget items of Ministry of Agriculture and Rural Affairs (grant number 20213578).

\begin{abstract}
Fatty acids in avocado fruit (Persea americana Mill.) are vital composition affecting flavour and nutritive value. Hence, horticulturalists are interested in illustrating the functions of transcription factors on fatty acid accumulation in avocado fruit. In the present study, the APETALA2/ethylene-responsive transcription factor gene, PaRAP2.1, was cloned from avocado mesocarp, and the subcellular localization demonstrated that PaRAP2.1 was located in the cytoplasm and nucleus. The PaRAP2.1 was introduced into Arabidopsis thaliana by Agrobacterium-mediated transformation. Furthermore, $P a R A P 2.1$ were functionally verified its effect on fatty acid biosynthesis. Histological analyses of lipid droplets displayed that the striking difference in the lipid droplets in the mature seeds between PaRAP2.1-overexpressing transgenic and wild-type Arabidopsis thaliana lines were revealed based on confocal microscopy images. Subsequently, fatty acid analyses of PaRAP2.1-overexpressing Arabidopsis thaliana lines displayed the significantly higher contents of fatty acids than those in the wild-type plants. Meanwhile, expression amount of ten genes involving in fatty acid biosynthesis dramatically up-regulated in the mature seeds of PaRAP2.1-overexpressing lines than those of wild-type plants. These results provide a theoretical basis for future research in regard to the function of PaRAP2.1 on fatty acid biosynthesis.
\end{abstract}

Keywords: avocado, $P a R A P 2.1$, fatty acids

\section{Introduction}

Fatty acids are important components in plant (Ge et al., 2017, 2018). The fatty acid biosynthesis has been studied extensively, and these have expounded transcription factors that regulate fatty aicd biosynthesis in plants (Ge et al., 2021a, 2021b). Members of many transcription factor families involving APETALA2/ethylene responsive factor (AP2/ERF) superfamily have been found to be involved in regulation in fatty acid biosynthesis in plants (Yeap et al., 2017). The AP2/ERF transcription factors are a multifarious superfamily expressed in plants, and AP2/ERF members have the conserved DNA binding domain, namely the AP2 domain, that binds to the gene's promoter region to regulate expression (Zhang et al., 2020). They are classified into three separate groups: ERF, RAV, and AP2 families according to the repeat number in AP2 domain (Zhang \& $\mathrm{Li}, 2018$ ). Currently, with studies in dicotyledonous plants such as Ricinus communis (Xu et al., 2013), Ziziphus jujuba (Zhang \& Li, 2018), Arabidopsis thaliana (Xie et al., 2019), Dimocarpus longan (Zhang et al., 2020), monocotyledonous plants such as Phyllostachys edulis (Wu et al., 2015), and gymnosperm such as Taxus chinensis (Zhang et al., 2019), we present a more in-depth knowledge of the functions and classification of AP2/ERF members.

In our previous study, the $137 \mathrm{PaAP} 2 / E R F$ genes were identified in avocado, and then the expression patterns of them in five developmental stages of avocado mesocarp were presented according to transcriptome data (Ge et al., 2021a). Subsequently, two PaAP2/ERF genes (PaWRI2 and PaWRI1) belonging to AP2 subfamily and eight PaAP2/ERF genes (PaRAP2.1, PaERF023, PaERF102-4, PaRAP2.2-2, PaERF109-5, PaERF082-1, PaRAP2.2-3, and PaRAP2.4-2) belonging to ERF subfamily were highly transcribed during five developmental 
stages of avocado mesocarp, which might regulate the accumulation of fatty acids in the avocado mesocarp (Ge et al., 2021a). Furthermore, the PaWRI1, a AP2 subfamily member, was selected to carry out the transgenic functional analysis, and the result implied that PaWRII might contribute to fatty acid accumulation (Ge et al., 2021a). Similarly, most of the genes governing fatty acid synthesis are found to be regulated by WRII in many plants (Kong \& Ma, 2019).

However, neither one of $P a A P 2 / E R F$ genes belonging to ERF subfamily has been found to modulate the vital genes participating in the fatty acid biosynthesis until now. In our previous study, the eight $P a A P 2 / E R F$ genes belonging to ERF subfamily were considered to take part in fatty acid biosynthesis in avocado mesocarp, and the PaRAP2.1 was more abundantly transcribed than other sever genes (Ge et al., 2021a). Therefore, in this study, we first chose PaRAP2.1, and performed the cloning and subcellular localization of PaRAP2.1. Second, to further exploit potential function of PaRAP2.1 on fatty acid accumulation, PaRAP2.1-overexpressing transgenic $A$. thaliana were developed, after which gene expression, lipid droplet observation, and targeted fatty acids detection of the transgenic $A$. thaliana and wild-type (WT) lines were carried out to analyse the contents of fatty acids. The data enriches our understanding in regard to the functions of PaRAP2.1 on fatty acid biosynthesis in the avocado mesocarp.

\section{Method}

\subsection{Plant Materials and Growth Conditions}

Avocado fruits (cultivar 'Hass') were collected from six 10-year-old trees in September 2018 at the Chinese Academy of Tropical Agricultural Sciences. Arabidopsis thaliana wild-type Col-0 seeds were disinfected surfaces with $70 \%$ ethanol for $30 \mathrm{~s}$ and $15 \%$ sodium hypochlorite for $15 \mathrm{~min}$, and then rinsed with distilled water three times for $20 \mathrm{~s}$. Then, the seeds were removed moisture from the surface, and placed on Murashige and Skoog medium.

\subsection{RNA Extraction and cDNA Synthesis}

The total RNA was extracted from avocado mesocarps and $A$. thaliana seeds. The mRNA was extracted from total RNA using poly-T oligo-attached magnetic beads. The first-strand cDNA was synthesized based on the sequence of the extracted RNA. The concentration of cDNA was diluted to $12.5 \mathrm{ng} / \mu \mathrm{L}$.

\subsection{Cloning of PaRAP2.1}

The coding sequence of $P a R A P 2.1$ is $1135 \mathrm{bp}$, and the amplification primer sequences of PaRAP2.1 were:

\section{5': TCTGATCAAGAGACAGGATCCATGGAGGGCACCGCCGCTCC}

\section{3': CATCGGTGCACTAGTGTCGACTAAATGCCCCATTTGCATCT}

PCR amplification system: synthetase $1 \mu \mathrm{L}, 2 \times$ PCR buffer $20 \mu \mathrm{L}$, dNTP Mixture $8 \mu \mathrm{L}, 5^{\prime}$ primer $0.3 \mu \mathrm{L}, 3^{\prime}$ primer $0.3 \mu \mathrm{L}$, cDNA $500 \mathrm{ng}$, plus $\mathrm{ddH}_{2} \mathrm{O}$ up to $50 \mu \mathrm{L}$. PCR amplification process: Initialized at $95{ }^{\circ} \mathrm{C}$ for 2 min and then 34 repeated cycles at $98{ }^{\circ} \mathrm{C}$ for $10 \mathrm{~s}, 60^{\circ} \mathrm{C}$ for $30 \mathrm{~s}$, and $68^{\circ} \mathrm{C}$ for $2 \mathrm{~min}$, with a final extension at $68{ }^{\circ} \mathrm{C}$ for $7 \mathrm{~min}$. PCR products were purified using an Axygen company Recovery Kit.

\subsection{Construction of PaRAP2.1 Transient Expression Vector and Subcellular Localization}

The vector plasmids sequenced correctly were transformed into Agrobacterium, spread on the plates including $25 \mathrm{mg} / \mathrm{L}$ kanamycin and $25 \mathrm{mg} / \mathrm{L}$ rifamycin. The monoclonal shaking bacteria were selected to grow overnight, the bacterial solutions were collected, and then resuspended in infiltration medium. Agrobacterium solutions containing vectors were blended in proportion. The liquid mixtures were transfused into leaves of tobacco for 28 days. After 3 days, the leaves of tobacco were scanned through confocal scanning microscope.

\subsection{Vector Construction and Plant Transformations}

To generate the PaRAP2.1-overexpressing (OE) construct, the full-length PaRAP2.1 CDSs was amplified and transferred into the pCAMBIA1300 vector including the $35 \mathrm{~S}$ promoter. We introduced the recombinant plasmids into Agrobacterium tumefaciens (GV3101). The floral dip method was used for genetic transformation of wild-type $A$. thaliana. Hygromycin-resistant plants were screened from transformed seeds, and then the $\mathrm{T}_{1}$ generation were obtained. $\mathrm{T}_{1}$ seeds were sown, and finally $\mathrm{T}_{3}$ transgenic $A$. thaliana plants were obtained.

\subsection{Quantitative Real Time PCR of Gene Expression}

The eight genes participating in fatty acid biosynthesis expressed in the seeds of the WT and PaRAP2.1-OE A. thaliana lines were chosen for qRT-PCR, and AtActin 7 was used as an endogenous control for normalizing data (Table 1). The qRT-PCR amplification process was described by Ge et al. (2019). Relative gene expression 
levels were calculated with the $2^{-\Delta \Delta \mathrm{Ct}}$ method (Livak \& Schmittgen, 2001). For each sample, the qRT-PCR analysis was completed with three biological replicates and two technical replicates.

Table 1. Primer sequence

\begin{tabular}{ll}
\hline Primer & Sequence information 5'-3' \\
\hline AtPDH $(E 1 \alpha) \mathrm{F}$ & ACTTCGCCAGCTTGTGATTC \\
AtPDH $(E 1 \alpha) \mathrm{R}$ & AAGATCGCTCCCTCTGACAG \\
AtACC $(C t \alpha) \mathrm{F}$ & TTCTTTACCACTGGACACCC \\
AtACC $(C t \alpha) \mathrm{R}$ & CTGCGACCTTAAAGGAACGC \\
AtACP4F & GAAGGTGTAGGGCGAAGACA \\
AtACP4R & GTGTAGACAGGCAGTGGACA \\
AtSADF & GGTATGTCGTCGCTTGTGAA \\
AtSADR & ACACGCTACTCACCTACACA \\
AtFATAF & ATGGCAGTTAGATTGGTGGG \\
AtFATAR & AGAGCCGAGTCGTTATGTCC \\
AtLACS $9 \mathrm{~F}$ & AGAGGGTTGAGGCGAAGAAC \\
AtLACS $9 \mathrm{R}$ & GTTGAAGCGAGCAGTGGAAC \\
AtGPAT1F & ATCACTCTCTTGAGCTGGCG \\
AtGPAT $1 \mathrm{R}$ & ATACTCCTCTGTGACGTGGC \\
AtFAD2F & TTGCTGGAAAGTGCTGACAA \\
AtFAD2R $2 \mathrm{R}$ & CTATGAGTTGGTCTCGCGAG \\
AtActin $7 \mathrm{~F}$ & TGCCCAGAAGTTCTATTCCAGC \\
AtActin $7 \mathrm{R}$ & CATAGTTGAACCACCACTGAGGAC \\
\hline
\end{tabular}

\subsection{Analysis of Fatty Acid Compositions by Gas Chromatography-Mass Spectrometry}

The fatty acid compositions of the A. thaliana seeds from the WT and PaRAP2.1-OE plants were determined by gas chromatography-mass spectrometry (GC-MS) as described by Ge et al. (2019). The oils extracted from the seeds of the WT and PaRAP2.1-OE $(20 \mu \mathrm{L})$ were saponified at $80^{\circ} \mathrm{C}(30 \mathrm{~min})$. After cooling, the solutions were mingled with $3 \mathrm{~mL} \mathrm{BF}-\mathrm{MeOH}(14 \%)$ and incubated at $75{ }^{\circ} \mathrm{C}(30 \mathrm{~min})$ to generate fatty acid methyl esters (FAMEs). The analyses were performed through an Agilent 7890B-7000B GC-MS with a DB-5MS column. The FAMEs were identified by comparing the retention times of the peaks with those of commercial standards and comparing the respective ion chromatograms with those in the NIST 2011 library. Methyl nonadecanoate was added as an internal standard and the FAMEs were quantified based on the calibration curves for the standards $\left(R^{2} \geq 0.995\right)$. The FAME contents $(\mathrm{mg} / 100 \mathrm{~g}$ fresh weight $)$ are herein presented as the mean \pm standard deviation of three biological replicates, each with two technical replicates.

\subsection{Histological Analyses}

To visualize the lipid droplets in the mature seeds from the WT and PaRAP2.1-OE A. thaliana plants, the method of sample handling and lipid droplet observation was described by Ge et al. (2019).

\section{Results and Discussion}

\subsection{Cloning and Subcellular Localization Analysis of PaRAP2.1}

Using the melon cDNA as a template, the fragment of PaRAP2.1 was amplified and analyzed. Band 1 was about $1131 \mathrm{bp}$ according to the DNA marker (DL2000) (Figure 1), which was consistent with the anticipative result of the present study. Through laser scanning microscopy, it was observed that PaRAP2.1 was located in the nucleus and cytoplasm of tobacco leaves. However, as shown in Figure 2, the green fluorescent signal of PaRAP2.1-GFP was mainly concentrated on the plasma membrane. These results indicated that the PaRAP2.1 might be a transcription factor that played a role in the nucleus and cytoplasm. However, subcellular localization shows that BnWRI1, the same AP2/ERF transcription factor, is only distributed in the nucleus (Wu et al., 2014; Li et al., 2015). 


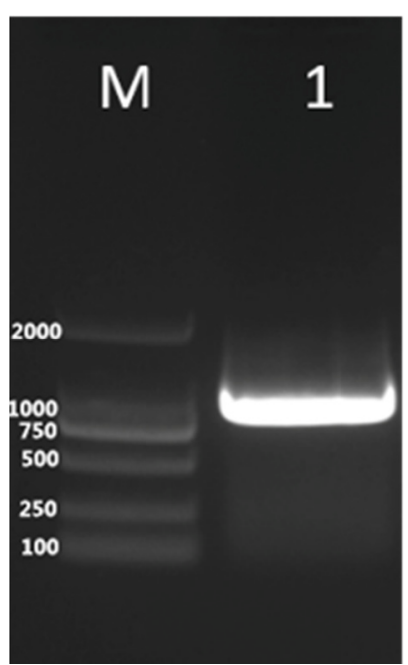

Figure 1. PCR amplification results of PaRAP2.1. M: TaKaRa company DL2000; 1: full length of PaRAP2.1

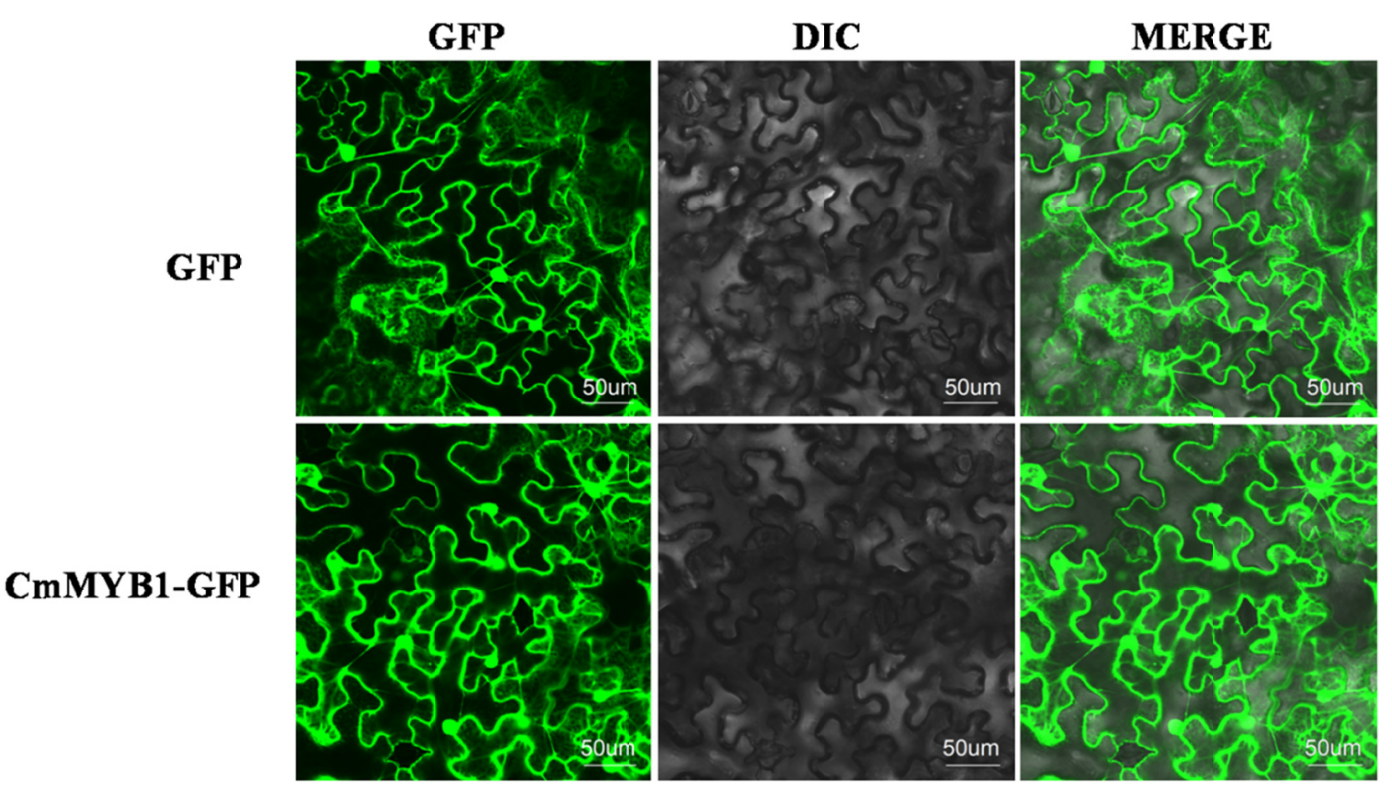

Figure 2. Subcellular localization of PaRAP2.1 in N. benthamiana leaves. PaRAP2.1 fused to the N-terminal of GFP under the control of the $35 \mathrm{~S}$ promoter. Bars $=50 \mu \mathrm{m}$

\subsection{The Effect of PaRAP2.1 on the Transcript Level of Ten Genes in Fatty Acid Synthesis and Lipid Assembly}

The leaves of transgenic $A$. thaliana were grown for about 4 weeks, the genome was extracted, and the target fragment was amplified. The expression of the PaRAP2.1 was detected in transgenic A. thaliana and positive controls, indicating that the PaRAP2.1 was integrated into the chromosome of $A$. thaliana (Figure 3). The phenotypic characteristic of transgenic $A$. thaliana $\mathrm{T}_{3}$ lines and wild-type diaplayed less variation during growth stage (Figure 4). However, in our previous study, the precocious flowering is simultaneously found in PaWRI1-OE and PaWRI2-OE transgenic tomato plants (Ge et al., 20121a). Similarly, BnWRI1 in transgenic Brassica napus plants also accelerate flowering and enhance oil accumulation in both seeds and leaves (Li et al., 2015). Besides, all AtWRII-OE transgenic potato plants demonstrate alterant tuber morphology, such as extended tuber, deeper eyes, and weight increment (Hofvander et al., 2016). These results suggested that the PaAP2/ERF genes presented the diverse biological function although PaRAP2.1, PaWRI1, and PaWRI2 all belong to AP2/ERF superfamily. 


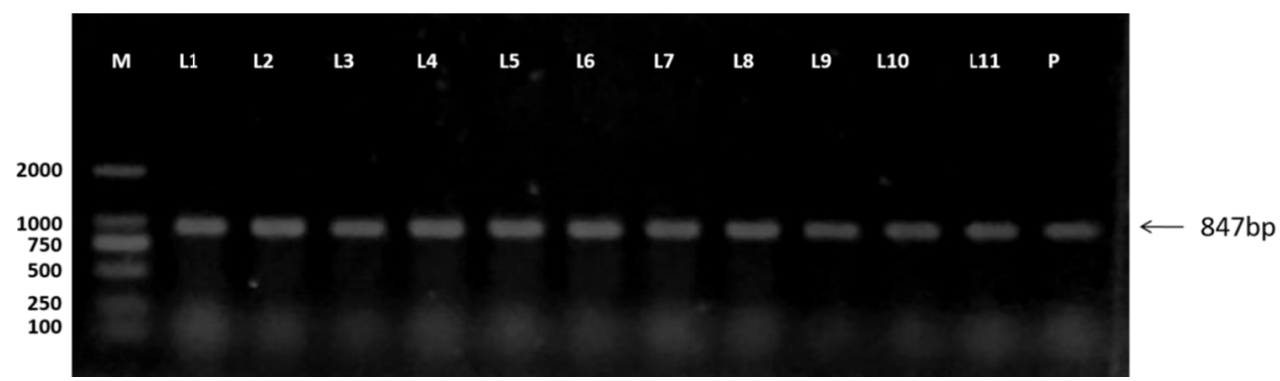

Figure 3. The detection of PaRAP2.1 expression in transgenic A. thaliana. M: DL2000; L1-L11: PaRAP2.1-overexpressing transgenic $A$. thaliana $\mathrm{T}_{3}$ different lines; P: pCAMBIA1300-35s-myc

A.

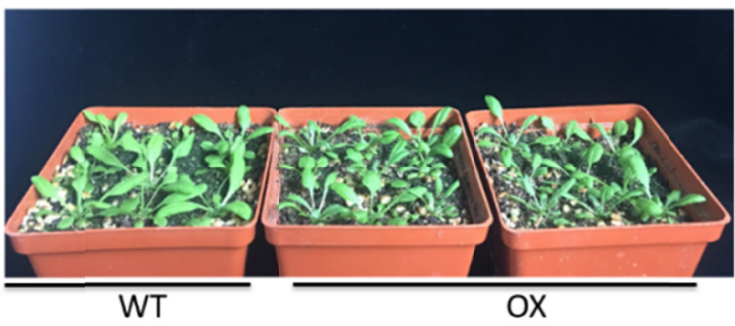

B.

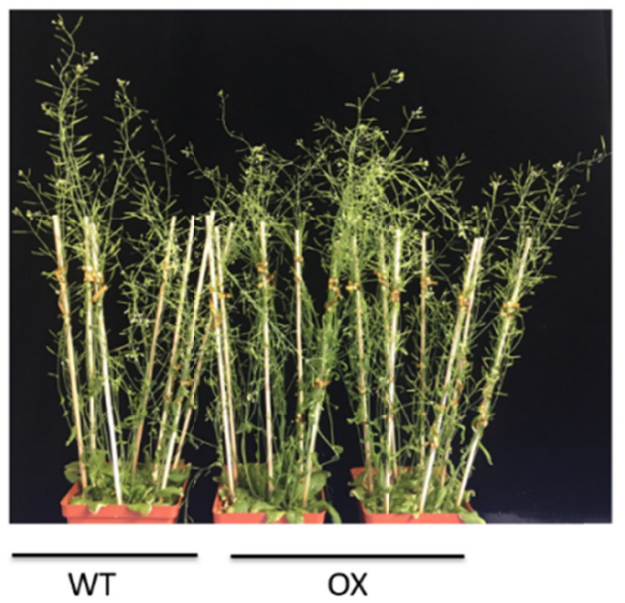

Figure 4. Phenotype analysis of transgenic A. thaliana. WT: wild-type Col-0; OX: PaRAP2.1 overexpression transgenic $A$. thaliana $\mathrm{T}_{3}$ different lines. A: Wild-type and overexpression $A$. thaliana vegetative growth conditions. B: Growth status of wild-type and overexpressing $A$. thaliana during reproductive growth period

The eight genes participating in fatty acid biosynthesis in PaRAP2.1-OE and WT plants were selected for qRT-PCR. The results indicated that the five out of eight genes in fatty acid biosynthesis were all up-regulated in PaRAP2.1-OE plants than in the WT, and the expression levels of AtPDH(E1 $)$, AtACP4, AtSAD, and AtFAD2 in PaRAP2.1-OE plants were more than twice as much as that of the WT (Figure 5). Recent report demonstrates that many genes in fatty acid biosynthesis are targets of another AP2/ERF transcription factor, WRI1 (Kong and Ma, 2019). In our previous study, transcriptome data of PaWRII-OE transgenic tomato plants indicates that 12 and five unigenes participating in fatty acid biosynthesis are up-regulated in PaWRI1-OE plants than in the WT (Ge et al., 2021a). The transcript amounts (> 100 FPKM) of genes in fatty acid biosynthesis including PDH(E3), $A C P 1$, two $F a t B$ paralogs, $S A D, A C C(C t \alpha)$, and $F A D 2$ are up-regulated the most, and the expression amounts in PaWRI1-2-OE plants are more than twice as much as that of the WT (Ge et al., 2021a). Similarly, several genes contributing to fatty acid biosynthesis, such as $A C P, P D H(E 1 \alpha), E A R, A C C(C t \alpha), L A C S O$, and $S A D$, are up-regulated in WRI1-OE plants than wild plants in some crops, such Zea mays (Pouvreau et al., 2011), B. napus (Li et al., 2015), Nicotiana benthamiana (Grimberg et al., 2015), Solanum tuberosum (Hofvander et al., 2016), Saccharum officinarum (Zale et al., 2016), and A. thaliana (Adhikari et al., 2016; Yeap et al., 2017). Besides, WRI5a, a AP2-domain transcription factor, is also considered as a master regulator of lipid biosynthesis by 
controlling lipid transfer and periarbuscular membrane formation in Medicago truncatula (Jiang et al., 2018). It was suggested that PaRAP2.1 was like PaWRI1 and WRI5a, and have a role in oil accumulation, which was agreement with the speculative result (Jiang et al., 2018; Ge et al., 2021a).
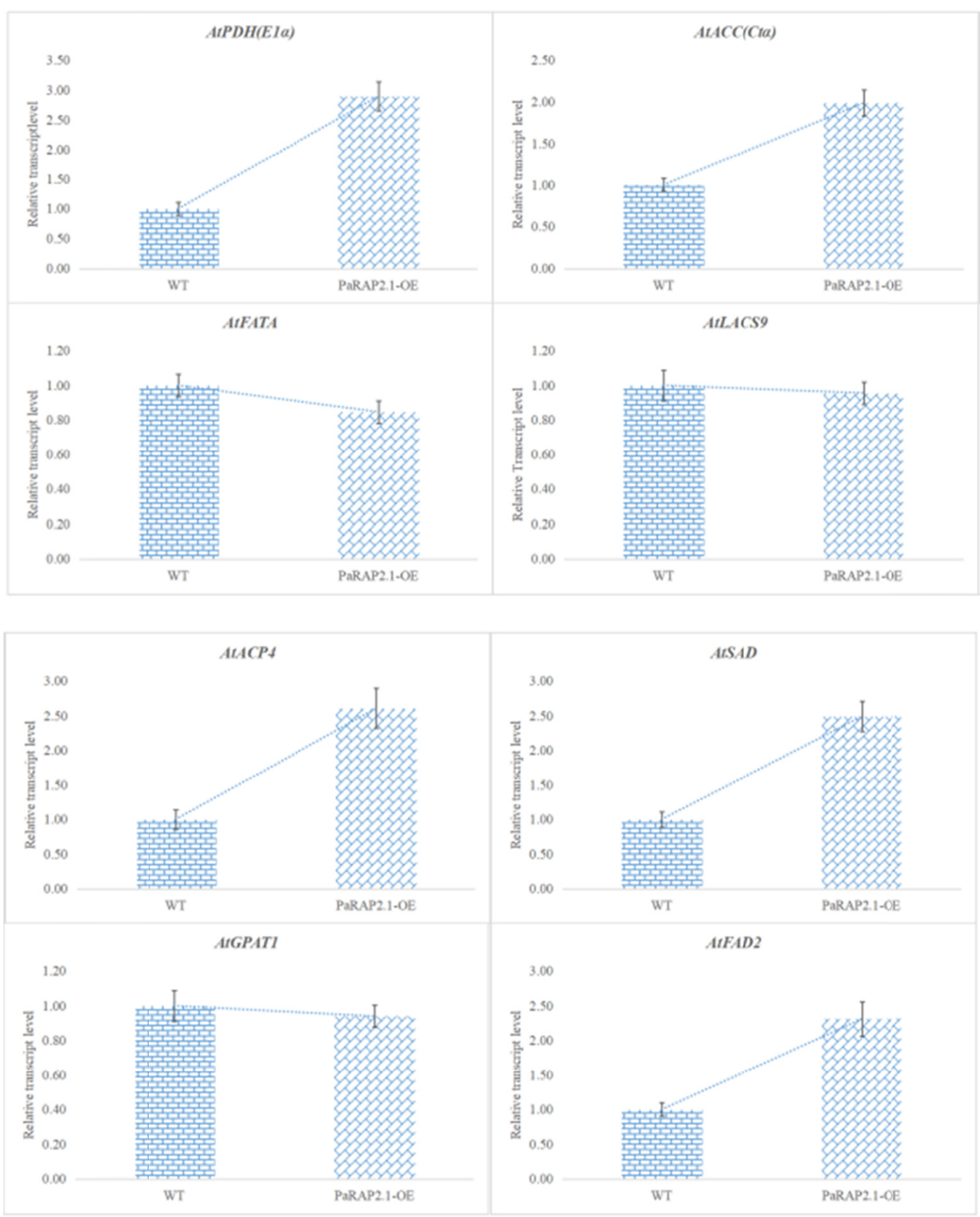

Figure 5. Eight genes were used for qRT-PCR in PaRAP2.1-OE and WT plants. The $2^{-\Delta \Delta \mathrm{Ct}}$ in WT plants were used as the control for normalization. Results represent the mean of three biological replicates and two technical replicates (mean $\pm \mathrm{SD}, n=6$ )

\subsection{Effects of PaRAP2.1 on Fatty Acid Contents}

To study the influence of PaRAP2.1 on fatty acid synthesis, six dominating of fatty acid compositions (palmitic, stearic, oleic, linoleic, Linolenic, and arachidic acids) were detected by GC-MS in PaRAP2.1-OE and WT plants. In the present study, the total fatty acid content in the PaRAP2.1-OE plants was more than $84 \%$ higher than that in the WT plants. All six critical fatty acid compositions in the PaRAP2.1-OE lines were higher than those in the WT lines, with the content of linoleic approximately 3-times higher in the PaRAP2.1-OE lines than in the WT liness (Figure 6). These results indicated that the expression of PaRAP2.1 might promote fatty acid accumulation. The complete ion chromatograms for the FAMEs of PaRAP2.1-OE and WT plants are provided in Figure 7. 
Some previous transgenic studies show that another AP2/ERF transcription factor, WRI1, could also induce effectively fatty acid biosynthesis in transgenic plants. In our previous study, the content of total fatty acid in the PaWRI1-2-OE transgenic tomato plants is more than 49\% higher than that in the untransformed controls, with oleic and linoleic acid contents nearly two fold higher in the PaWRI1-2-OE lines than in the WT lines (Ge et al., 2021a). Similarly, a GC-MS analysis of transgenic Brassica napus lines displays that the content of total fatty acid in the leaves of BnWRI1-OE plants is almost 53\% higher than that in the WT plants, with most of this difference due to the greater abundance of oleic, palmitic, linoleic, and stearic acids in the transgenic plants (Li et al., 2015). In a previous study, the total fatty acid content is reportedly 9\% higher in BnWRI1-OE transgenic Brassica napus plants than that in the WT plants, with the contents of oleic, linolenic, stearic, linoleic, and palmitic acids dramatically higher in the BnWRI1-OE transgenic plants than that in the untransformed controls (Wu et al., 2014). In another investigation involving a GC-MS analysis, the fatty acid content of transgenic maize lines is approximately one-third fold higher than that in the untransformed controls (Shen et al., 2010).

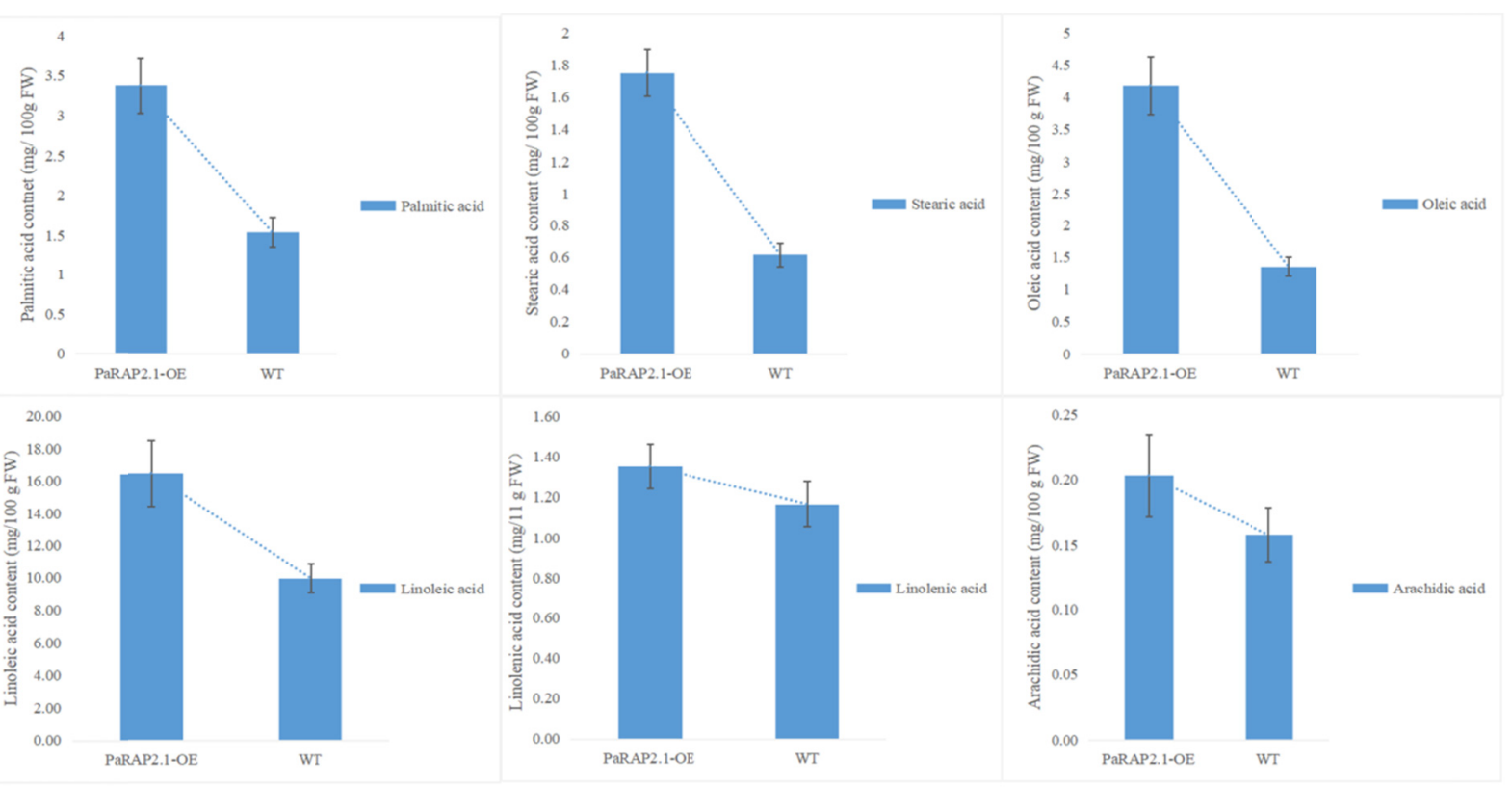

Figure 6. Fatty acid compositions of the mature seeds in the PaRAP2.1-OE and WT plants. The fatty acid contents $(\mathrm{mg} / 100 \mathrm{~g} \mathrm{FW})$ were herein presented as the mean \pm standard deviation of three biological replicates, each with two technical replicates 

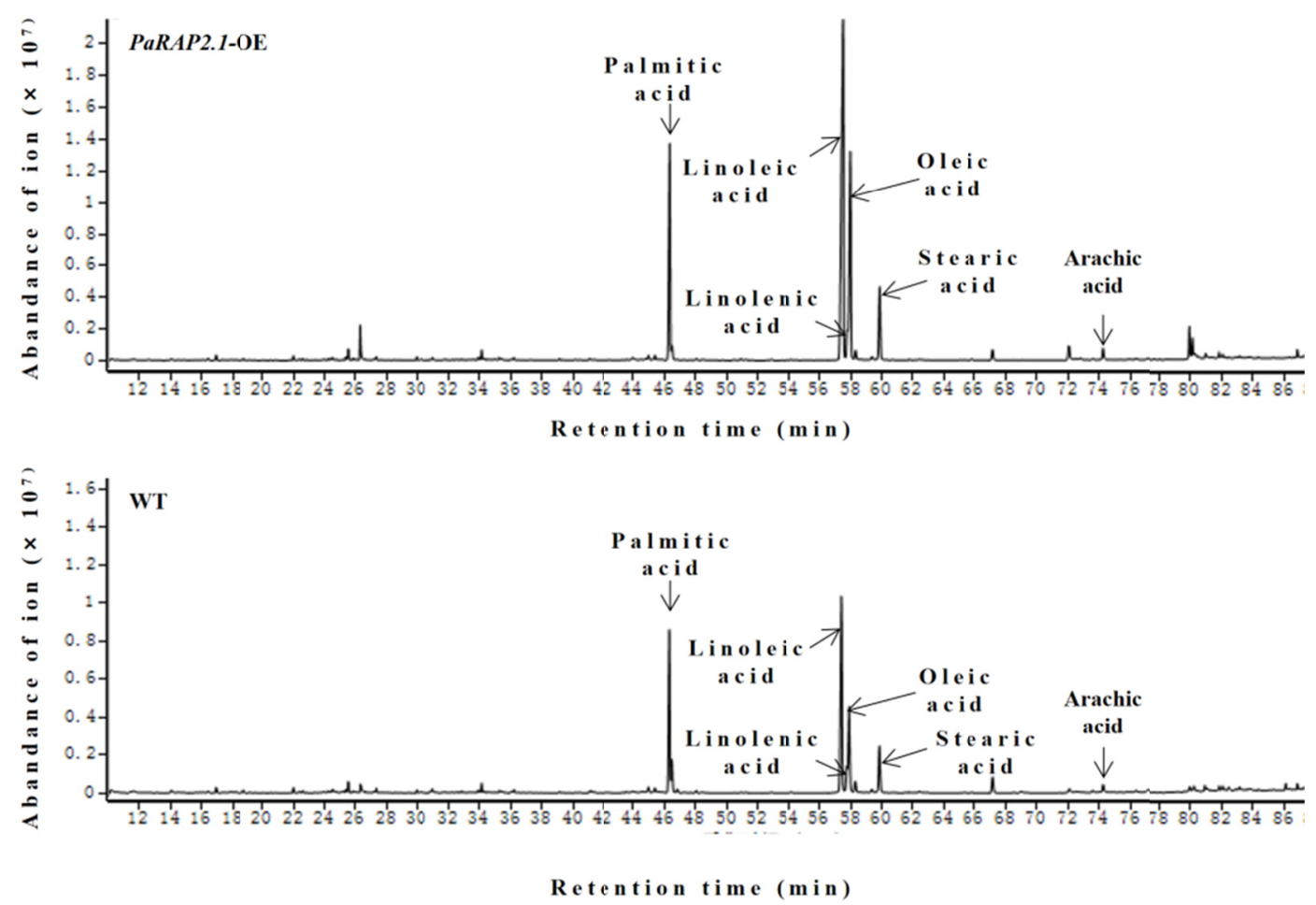

Figure 7. Total ion chromatogram of the fatty acid methyl esters of the PaRAP2.1-OE and WT plants

\subsection{Histological Analyses of Lipid Droplets in PaRAP2.1-OE and WT Plants}

In our previous studies, the total fatty acid content is always positively correlated with the area and number of lipid droplets (Ge et al., 2019, 2021b). Therefore, in the present study, the lipid droplets in the mature seeds of PaRAP2.1-OE and WT plants were visualized though a histological analysis. A great many lipid droplets (Figure 8B) had formed in the mature seeds of PaRAP2.1-OE plants, occupying most of the cell volume. In contrast, a few lipid droplets (Figure 8A) were founded at the edge of the cell wall in the seed of WT plants. Our histological analyses verified the observably difference in total fatty acid content measured though GC-MS between PaRAP2.1-OE and WT plants in the present study. Similarly, the accumulation of lipid droplets in AtWRI1-OE transgenic sugarcane leaf tissue is remarkable relative to WT plants (Zale et al., 2016).
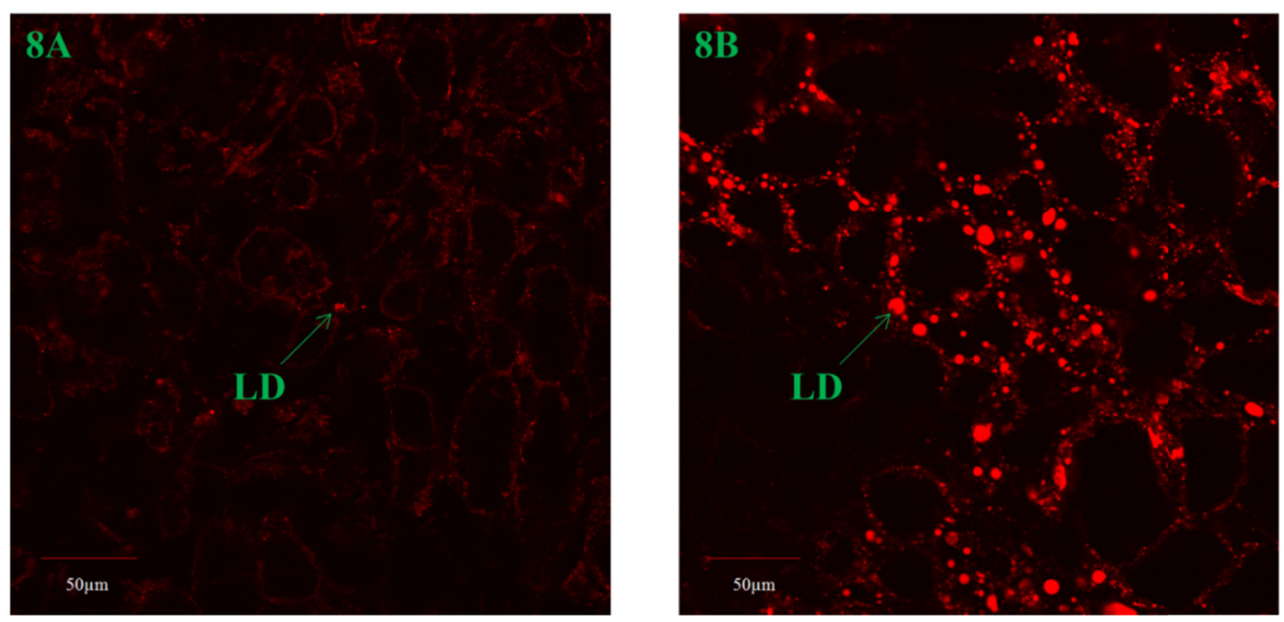

Figure 8. Confocal microscopy images of lipid droplets in WT (A) and PaRAP2.1-OE plants (B) using Nile red staining. LD: lipid droplet 


\section{Conclusion}

The functional analyses of transgenic A. thaliana lines overexpressing PaRAP2.1 illustrated the effects of the encoded transcription factors on fatty acid biosynthesis. The data suggested that PaRAP2.1 might be conducive to fatty acid biosynthesis. The results described herein may help to demonstrate the involvement of PaRAP2.1 in the fatty acid biosynthetic pathways in plants. The produced data offer worthy clues in regard to the biological functions of AP2/ERF transcription factors in plants.

\section{References}

Adhikari, N. D., Bates, P. D., \& Browse, J. (2016). WRINKLED1 rescues feedback inhibition of fatty acid synthesis in hydroxylase-expressing seeds. Plant Physiology, 171, 179-191. https://doi.org/10.1104/ pp.15.01906

Ge, Y., Dong, X. S., Liu, Y. Z., Yang, Y., \& Zhan, R. L. (2021b). Molecular and biochemical analyses of avocado (Persea americana) reveal differences in the oil accumulation pattern between the mesocarp and seed during the fruit developmental period. Scientia Horticulturae, 276, $109717 \mathrm{https} / / / \mathrm{doi} .0 \mathrm{rg} / 10.1016 /$ j.scienta.2020.109717

Ge, Y., Dong, X. S., Wu, B., Xu, Z. N., Zhou, Z. X., Lin, X. E., ... Ma, W. H. (2019). Physiological, histological, and molecular analyses of avocado mesocarp fatty acids during fruit development. Journal of Agricultural Science, 11, 1-10. https://doi.org/10.5539/jas.v11n1p1

Ge, Y., Si, X. Y., Cao, J. Q., Zhou, Z. X., Wang, W. L., \& Ma, W. H. (2017). Morphological characteristics, nutritional quality, and bioactive constituents in fruits of two avocado (Persea americana) varieties from hainan province, China. Journal of Agricultural Science, 9, 8-17. https://doi.org/10.5539/jas.v9n2p8

Ge, Y., Si, X. Y., Wu, B., Dong, X. S., Xu, Z. N., \& Ma, W. H. (2018). Oil content and fatty acid composition of the seeds of 16 avocado (Persea americana) accessions collected from southern China and their application in a soap bar. Journal of Agricultural Science, 10, 69-78. https://doi.org/10.5539/jas.v10n11p69

Ge, Y., Zang, X. P., Yang, Y., Wang, T., \& Ma, W. H. (2021a). In-depth analysis of potential PaAP2/ERF transcription factor related to fatty acid accumulation in avocado (Persea americana Mill.) and functional characterization of two PaAP2/ERF genes in transgenic tomato. Plant Physiology and Biochemistry, 158, 308-320. https://doi.org/10.1016/j.plaphy.2020.11.016

Grimberg, Å., Carlsson, A. S., Marttila, S., Bhalerao, R., \& Hofvander, P. (2015). Transcriptional transitions in Nicotiana benthamiana leaves upon induction of oil synthesis by WRINKLED1 homologs from diverse species and tissues. BMC Plant Biology, 15, 192. https://doi.org/10.1186/s12870-015-0579-1

Hofvander, P., Ischebeck, T., Turesson, H., Kushwaha, S. K., Feussner, I., Carlsson, A. S., \& Andersson, M. (2016). Potato tuber expression of Arabidopsis WRINKLED1 increases triacylglycerol and membrane lipids while affecting central carbohydrate metabolism. Plant Biotechnology Journal, 14, 1-16. https://doi.org/ $10.1111 /$ pbi.12550

Jiang, Y., Xie, Q. J., Wang, W. X., Yang, J., Zhang, X. W., Nan, Y., ... Wang, E. (2018). Medicago AP2-domain transcription factor WRI5a is a master regulator of lipid biosynthesis and transfer during Mycorrhizal symbiosis. Molecular Plant, 11, 1344-1359. https://doi.org/10.1016/j.molp.2018.09.006

Kong, Q., Yuan, L., \& Ma, W. (2019). WRINKLED1, a "Master Regulator" in transcriptional control of plant oil biosynthesis. Plants, 8, 238. https://doi.org/10.3390/plants8070238

Li, Q., Shao, J. H., Tang, S. H., Shen, Q. W., Wang, T. H., Chen, W. L., \& Hong, Y. Y. (2015). Wrinkled1 accelerates flowering and regulates lipid homeostasis between oil accumulation and membrane lipid anabolism in Brassica napus. Frontiers in Plant Science, 6, 1015. https://doi.org/10.3389/fpls.2015.01015

Livak, K. J., \& Schmittgen, T. D. (2001). Analysis of relative gene expression data using real-time quantitative PCR and the $2^{-\Delta \Delta C T}$ method. Methods, 25, 402-408. https://doi.org/10.1006/meth.2001.1262

Pouvreau, B., Baud, S., Vernoud, V., Morin, V., Py, C., Gendrot, G., ... Rogowsky, P. M. (2011). Duplicate maize Wrinkled 1 transcription factors activate target genes involved in seed oil biosynthesis. Plant Physiology, 156, 674-686. https://doi.org/10.1104/pp.111.173641

Shen, B., Allen, W. B., Zheng, P. Z., Li, C. J., Glassman, K., Ranch, J., ... Tarczynski, M. C. (2010). Expression of ZmLEC1 and ZmWRI1 increases seed oil production in maize. Plant Physiology, 153, 980-987. https://doi.org/10.1104/pp.110.157537 
Wu, H. L., Lv, H., Li, L., Liu, J., Mu, S. H., Li, X. P., \& Gao, J. (2015). Genome-wide analysis of the AP2/ERF transcription factors family and the expression patterns of DREB genes in Moso bamboo (Phyllostachys edulis). PLoS ONE, 10, e0126657. https://doi.org/10.1371/journal.pone.0126657

Wu, X. L., Liu, Z. H., Hu, Z. H., \& Huang, R. Z. (2014). BnWRII coordinates fatty acid biosynthesis and photosynthesis pathways during oil accumulation in rapeseed. Journal of Integrative Plant Biology, 56, 582-593. https://doi.org/10.1111/jipb.12158

Xie, Z. L., Nolan, T. M., Jiang, H., \& Yin, Y. H. (2019). AP2/ERF transcription factor regulatory networks in hormone and abiotic stress responses in Arabidopsis. Frontiers in Plant Science, 10, 228. https://doi.org/ $10.3389 /$ fpls.2019.00228

Xu, W., Li, F., Ling, L. Z., \& Liu, A. Z. (2013). Genome-wide survey and expression profiles of the AP2/ERF family in castor bean (Ricinus communis L.). BMC Genomics, 14, 785. https://doi.org/10.1186/1471-2164$14-785$

Yeap, W. C., Lee, F. C., Shan, D. K. S., Musa, H., Appleton, D. R., \& Kulaveerasingam, H. (2017). WRI1-1, ABI5, NF-YA3 and NF-YC2 increase oil biosynthesis in coordination with hormonal signaling during fruit development in oil palm. Plant Journal, 91, 97-113. https://doi.org/10.1111/tpj.13549

Zale, J., Jung, J. H., Kim, J. K., Pathak, B., Karan, R., Liu, H., ... Altpeter, F. (2016). Metabolic engineering of sugarcane to accumulate energy-dense triacylglycerols in vegetative biomass. Plant Biotechnology Journal, 14, 661-669. https://doi.org/10.1111/pbi.12411

Zhang, M., Chen, Y., Jin, X. F., Cai, Y. X., Yuan, Y. Y., Fu, C. H., \& Yu, L. J. (2019). New different origins and evolutionary processes of AP2/EREBP transcription factors in Taxus chinensis. BMC Plant Biology, 19, 413. https://doi.org/10.1186/s12870-019-2044-z

Zhang, S. T., Zhu, C., Lyu, Y. M., Chen, Y., Zhang, Z. H., Lai, Z. X., \& Lin, Y. L. (2020). Genome-wide identification, molecular evolution, and expression analysis provide new insights into the APETALA2/ethylene responsive factor (AP2/ERF) superfamily in Dimocarpus longan Lour. BMC Genomics, 21, 62. https://doi.org/10.1186/s12864-020-6469-4

Zhang, Z., \& Li, X. A. (2018). Genome-wide identifcation of AP2/ERF superfamily genes and their expression during fruit ripening of Chinese jujube. Scientific Reports, 8, 15612. https://doi.org/10.1038/ s41598-018-33744-w

\section{Copyrights}

Copyright for this article is retained by the author(s), with first publication rights granted to the journal.

This is an open-access article distributed under the terms and conditions of the Creative Commons Attribution license (http://creativecommons.org/licenses/by/4.0/). 